\title{
Design Analysis of External Automatic Leveling Mechanism in Radars
}

\author{
Shi-lei Zhi, Run-ze Yang \\ Mechanical Manufacturing and Automation Lab, Mechanical Engineering College, Shijiazhuang, \\ 050003, China
}

Keywords: external, leveling mechanism, ball screw, self-lock, single direction non-return bearing

\begin{abstract}
Most of radar apparatus equipped from the 1980s to the early part of this century were mainly manual and hydraulic leveling which were time-consuming and of low leveling precision. Thus, to avoid these problems, this paper attempts to design a leveling mechanism, which is driven by AC servo motor and supported by ball screw with high transmission efficiency and carrying capacity. Meanwhile, a new method of braking and self-locking composed of motor braking and single direction non-return bearing is introduced in the paper. This leveling mechanism with the characteristics of small volume, simple structure and high efficiency is of universality and portability.
\end{abstract}

\section{Introduction}

In recent years, local war under modern high-tech conditions has a growing demand to the radar mobility which is a reflection of the ability to rapidly conceal and relocate. Generally, the requirements of radar mobility include erect-fold and leveling time, the number of operating personnel and delivery unit, position adaptability etc.[1]. For this reason, the problem associated with radar leveling has been of interest to investigators for a long time, and increasing attention has been paid in recent years to the development of the reliable and automatic erect-fold and leveling radar apparatus, which is beneficial to the improvement of radar mobility.

Currently, all the new radar apparatus have automatic erect-fold and leveling function, as well as high mobility. However, from the 1980s to the early part of this century, most of radar apparatus were mainly manual and hydraulic leveling. The manual leveling is the earliest way by which the operators adjust a screw again and again through observing the bulb of level. This method is timeconsuming and the leveling precision is not high. In recent years, the automatic leveling methods have been developed very quickly, including hydraulic leveling and mechatronic leveling system. The hydraulic leveling has many advantages compared with the manual leveling, which includes heavier load, higher precision and shorter time. However, there are many shortcomings for the hydraulic system, such as low sensitivity, long respond time, difficult maintaining, high cost and oil leaking [2]. Obviously, these methods cannot meet the current requirements for information under the condition of power-driven warfare, and severely reduce the radar battlefield survivability. There exist some prominent problems such as time-consuming, more operators and lower precision.

In order to solve the problems described above, another automatic leveling method is discussed in this paper. The mechatronic leveling method has many advantages compared with other leveling methods. For example, the required time is very short, action is more sensitive, precision and reliability is higher, and maintenance is easier [3]. It is the particular intention of this paper to remark upon the design of erect-fold and leveling mechanism for the improvement of radar mobility.

\section{Structure design of leveling mechanism}

Leveling mechanism is an important part of leveling system. The radar vehicle and leveling system is shown in Fig.1, which is composed of leveling mechanism, tilt sensor, controller and motor driver. 


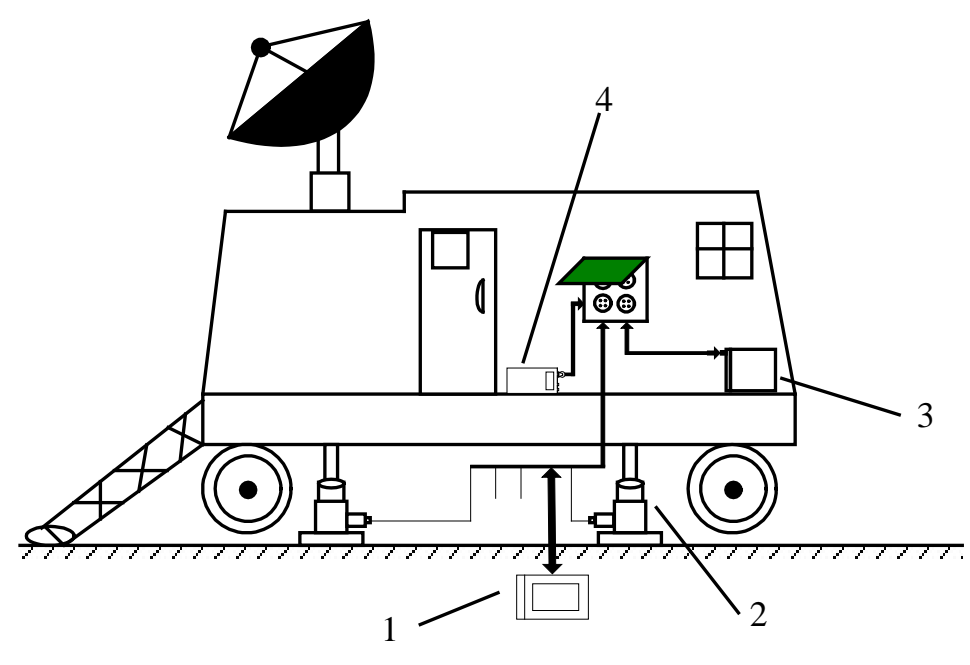

1. operation control terminal, 2. support and leveling unit,

3. motor driver and control unit, 4. tilt sensor

Fig.1: The diagrammatic sketch of leveling system

The working principle of automatic leveling system is shown in Fig.2. When the system is working, the communication port will transport vehicle angle signal which is detected by tilt sensor to controller. Then, the motor is driven by controller to control the lifting and drop of legs, and maintain the level of vehicle platform lastly.

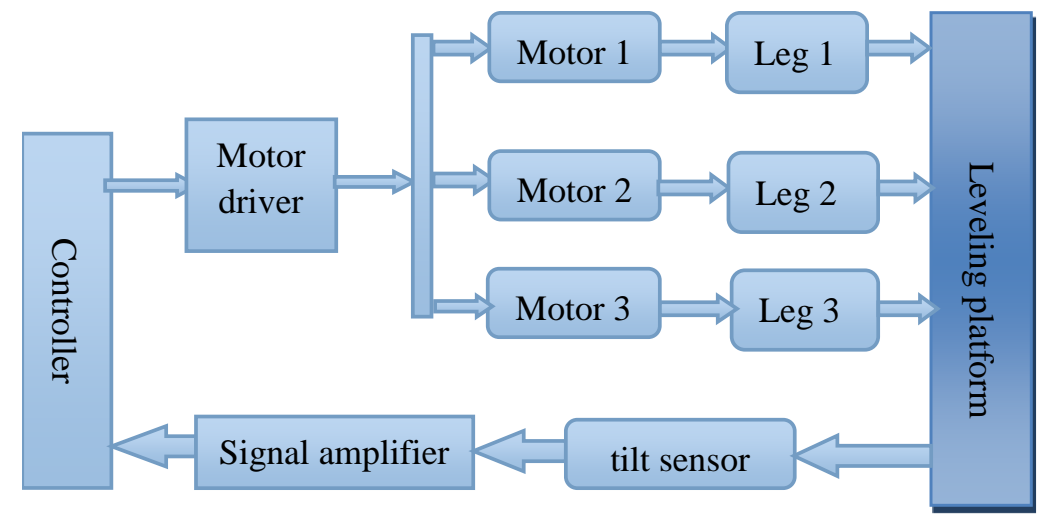

Fig.2: The process of leveling

\subsection{Constitution and working principle of mechanism}

As Fig.3 shows, the leveling mechanism is composed of servo motor, reducer, reduction gear, ball screw, lock mechanism and other components. Firstly, the motor reduces rotate speed through reducer and gear, then drives ball screw to rotate, when screw rotates, the screw nut moves up and down at the same time, accomplishing the rise and fall of vehicle. 


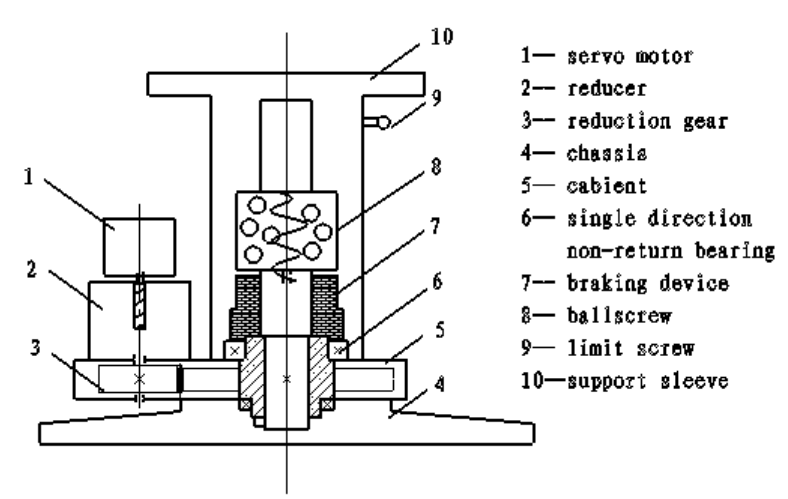

Fig.3: The constitution of leveling mechanism

\subsection{Design of main machinery component}

\subsubsection{Design and select ball screw}

i) Select screw.

According to the load and structure of vehicle, the maximum axial load of single leg is calculated. Then, the medium diameter of ball screw can be calculated according to axial strength as follows[4].

$$
d_{2} \geq 0.8(F /(\psi[p]))^{1 / 2}
$$

Where $\mathrm{d} 2$ is the medium diameter of screw, $\mathrm{F}$ is the maximum axial load of single leg, $\psi$ is the ratio of height and medium diameter of screw, and [p] is the allowable pressure of screw.

Meantime, according to the condition of losing stabilization, the bottom diameter of screw can be calculated [5]. As follows,

$$
d_{3} \geq \sqrt[4]{64 F K L_{y}^{2} \times 10^{-4} /\left(f_{k} \pi^{3} E\right)}
$$

Where, $d_{3}$ is the bottom diameter of screw, $K$ is the stability safety factor of screw, $f_{k}$ is the support factor of screw, E is the elastic modulus of screw, and Ly is the length of screw, chosen by structure of screw.

Considering both the two effects, to meet the actual needs of structure, the ball screw is chosen by Handbook of Mechanical Design.

ii) Life checking of screw

The life of screw can be calculated as follows [6].

$$
L_{h}=\left(C_{a} / F\right) \times 10^{6}(r)
$$

Where Ca is the dynamic load rating of screw, Lh is the life of screw, and generally the allowable life of screw is assigned the value $\mathrm{Lh}=5 \times 106$. Therefore, it will be accepted as long as the Lh is less than the allowable life.

iii) Stability checking of screw

The ball screw will be compressed by axial force, so the deflection of screw can be calculated as follows [5].

$$
\Delta L=\frac{F P}{E S}
$$

Where $S$ is the minimum cross-sectional area of screw, and $P$ is helical pitch of screw. It will be accepted as long as the deflection of screw is less than the allowable standard.

\subsubsection{Design of braking and self-locking device}

Radar vehicle should maintain a constant height and not be dropped after it rises to desired location, even if losing power. However, the ball screw has high transmission efficiency without self-locking function, so it need lock mechanism to prevent inverse driving with power interruption. This paper applies motor braking to function together with single direction non-return bearing 
which cooperates with frictional damping device[7], as is shown in Fig.3.

The brake has no effect when motor is working, but once the motor loses power, the brake immediately achieves mechanical brake, to prevent decline of vehicle. In the meantime, in order to play a dual role in ensuring the safety of radar at work, this paper adds single direction non-return bearing which only allows one-way rotating, reverse braking in the view of structure design.

\subsubsection{Design other part of device}

1) Choose servo motor

Step1: According to design requirement, the axial movement speed of screw is known, so the rotation rate of screw can be calculated.

$$
n_{0}=60 \mathrm{~V} / \mathrm{S}(\mathrm{r} / \mathrm{min})
$$

Step2: According to the axial load and movement speed of screw, the minimum output power of screw can be calculated.

$$
P_{\text {min }}=N_{2} V
$$

Step3: The transmission efficiency of leveling mechanism is known as follows.

$$
\eta=\eta_{1} \eta_{2} \eta_{3}
$$

Where, $\eta_{1}$ is the transmission efficiency of screw, $\eta_{2}$ is the transmission efficiency of reducer system, $\eta_{3}$ is the transmission efficiency of other part.

Thus, the minimum working power of motor can be calculated.

$$
P_{\text {omin }}=P_{\min } / \eta
$$

Lastly, the motor can be chosen by $n_{0}$ and $P_{0 \min }$.

2) Design of reducer system

In order to meet the design requirements, it needs to add the reducer system between the motor and ball screw, the planetary gear reduction and cylindric spur gear are applied in this leveling mechanism. The rotation rate of motor and screw are defined as $n_{1}, n_{0}$, so transmission ratio can be calculated as follows.

$$
i=n_{1} / n_{0}
$$

Thus, the reducer system can be designed by distributing the transmission ratio.

\section{The realization of erect-fold and leveling}

This mechanism has semi-automatic and automatic modes which are used for erect-fold and leveling, the main mode is automatic leveling. The work is carried out in three major sections, fig. 4 shows the flow chart of erect-fold and leveling.

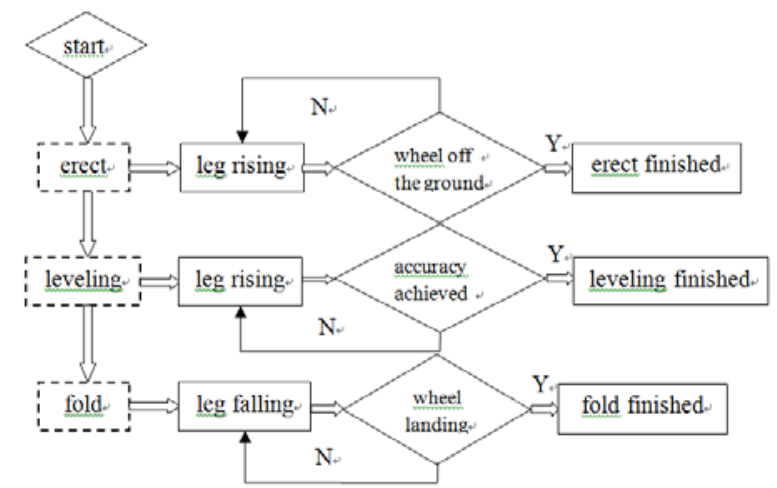

Fig. 4: The flow chart of erect-fold and leveling 


\section{Conclusions}

The design and application of external and independent automatic leveling mechanism can not only shorten the time of preparation and erect-fold for the early radar, but also improve the mobility of radar fundamentally. This article focuses on the structural analysis and design of erect-fold and leveling mechanism. The ball screw driven by servo motor has high transmission efficiency and bearing capacity, and the innovation lies in the combination of motor braking and single direction non-return bearing function to achieve braking and self-locking. Practices show that it has many advantages over common overrunning clutch, such as compact structure, high reliability and flexible mobility. However, due to paper length limited, the electric control of leveling device is not involved in this paper. Furthermore, the mechanical structure needs to be further optimized, so that it has a broader universality and portability in a variety of conditions.

\section{References}

[1] Chu Xin-Feng, Primary Study on Automatic Leveling and Lifting System for Vehicle-borne Radars, Wuhan: Huazhong University of Science and Technology, pp. 1-2, 2007.

[2] Lu Chao-Shuang, Research on Leveling Control System for Electromechanical Vehicle-borne Radars Platform with high accuracy, Chengdu: University of Electronic Science and Technology of China, pp. 1-2, 2007.

[3] Zhou Chun-Yan, The Development and Design of Automatic leveling Control System Based on PLC, Xi'an: Xidian University, pp. 6-8, 2010.

[4] Li Guo-Zhu, Mechanical Design and Theory, Beijing: Science Press, 2003.

[5] Zhang Jian-Ming, Mechanical-electrical integration system design, Beijing: Beijing Institute of Technology Press, pp. 35, 2007.

[6] Yang Li-Ming, The Handbook of Mechanical-electrical integration system design, Beijing: National Defence of Industry Press, 1997.

[7] Li Ming, Li Quan-Qing, Development of 3D CNC Engraving and Milling Machne.Machine tool \& Hydraulics, 35(2), pp. 95-98, February 2007. 